Marquette University

e-Publications@Marquette

Psychology Faculty Research and Publications

Psychology, Department of

$1-1-2012$

Behavioral Factors Influencing Health Outcomes in Youth with Type 1 Diabetes

Jessica C. Kichler

Medical College of Wisconsin

Ashley Moss

Marquette University, ashley.moss@marquette.edu

Astrida S. Kaugars

Marquette University, astrida.kaugars@marquette.edu

Published version. US Endocrinology, Vol. 8, No. 2 (Winter 2012): 77-83. Permalink. (C) 2012 Touch Medical Media. Used with permission. 


\title{
Behavioral Factors Influencing Health Outcomes in Youth with Type 1 Diabetes
}

\author{
Jessica C Kichler, PhD, CDE, ${ }^{1}$ Ashley Moss, BA² and Astrida S Kaugars, PhD $^{3}$ \\ 1. Associate Professor of Pediatrics, Cincinnati Children's Hospital Medical Center, Cincinnati, Ohio, US; 2. Student, Marquette University, \\ Milwaukee, Wisconsin, US; 3. Associate Professor of Psychology, Marquette University, Milwaukee, Wisconsin, uS
}

\begin{abstract}
The Pediatric Self-Management Model provides an overview of how behavioral factors influence children's chronic medical illnesses. This general framework is used to organize the present review of how self-management behaviors, contextual factors, and processes impact health outcomes for adolescent youth with type 1 diabetes. Adherence has been widely studied in the diabetes literature, and there are consistent findings demonstrating associations between aspects of self-management, adherence, and metabolic control, yet there are still equivocal approaches to adherence assessment methodology (e.g. global versus specific measures). Metabolic control is a hallmark health outcome for youth with type 1 diabetes, but additional outcomes need to be further explored. Future research should utilize the Pediatric Self-Management Model's operational definitions to guide empirically-supported interventions for youth with type 1 diabetes.
\end{abstract}

\section{Keywords}

Behavioral factors, type 1 diabetes, youth

Disclosure: The authors have no conflicts of interest to declare.

Received: September 11, 2012 Accepted: October 29, 2011 Citation: US Endocrinology, 2012;8(2):77-83

Correspondence: Jessica C Kichler, PhD, CDE, Cincinnati Children's Hospital Medical Center, Behavioral Medicine and Clinical Psychology, 3333 Burnet Ave, MLC 3015 ,

Cincinnati, OH 45229, US. E: jessica.kichler@cchmc.org

Adherence to medical regimen is often identified as a hallmark modifiable factor that impacts health outcomes for those with chronic medical illnesses. Rapoff suggests that adherence to medical regimens is estimated to be between $50-55 \%$ for all chronically ill patients. He describes a number of patient and family (e.g., demographic, adjustment/coping, family involvement), disease- and regimen-related correlates to adherence; however, correlates do not imply causation and should be interpreted cautiously. ${ }^{1}$ Researchers have rated non-adherence rates to be between 20 to $93 \%^{1-3}$ for youth with type 1 diabetes and have found that adherence is widely accepted as related to diabetes control. ${ }^{4}$

In a recent review article, Modi et al. make a distinction between two constructs: self-management (i.e., the interactions of health behaviors and related processes that patients and families engage in to care for chronic medical conditions) and adherence (i.e., the extent to which a person's behavior coincides with medical or health advice). ${ }^{5}$ In their Pediatric Self-Management Model, self-management influences adherence, which then affects outcomes, including health outcomes. The authors also suggest that there may be certain self-management factors that impact outcomes without the mediating role of adherence in pediatric conditions. ${ }^{5}$

This present review article will focus on the first two of three stages in the Pediatric Self-Management Model, self-management and adherence, to discuss the behavioral factors that may influence health outcomes for youth with type 1 diabetes during adolescence (see Figure 1). Findings from individual, family, peer, and group therapy interventions that have targeted these behavioral factors in order to improve health outcomes will then be outlined.

\section{Self-management}

Modi et al. describe the construct of self-management as having three components. ${ }^{5}$ The first component is self-management behaviors, which are the actual behaviors performed by the youth and/or family in order to care for a chronic medical illness. Secondly, there are contextual variables, which are four systems that impact how the self-management behaviors occur. These include individual, family, community, and healthcare domains. Finally, there are processes that link the self-management behaviors with the contextual systems. These processes include individuals' cognitive, emotional, and social perceptions. Three components of self-management, self-management behaviors, contextual variables, and processes as they relate to youth with type 1 diabetes, will be reviewed below (see Table 1).

\section{Self-management Behaviors}

Self-management behaviors, defined as examples of the 'neutral' behaviors that are employed to help manage a chronic medical illness, have been widely examined in youth with type 1 diabetes. Some examples of self-management behaviors that researchers have repeatedly identified as relevant to the management of diabetes are parental involvement and collaboration, the division of diabetes responsibility in the family and subsequent transfer of diabetes care during adolescence, and parent-youth communication. 


\section{Figure 1: The Pediatric Self-Management Model Applied to Behavioral Factors Associated with Health Outcomes in Youth with Type 1 Diabetes}

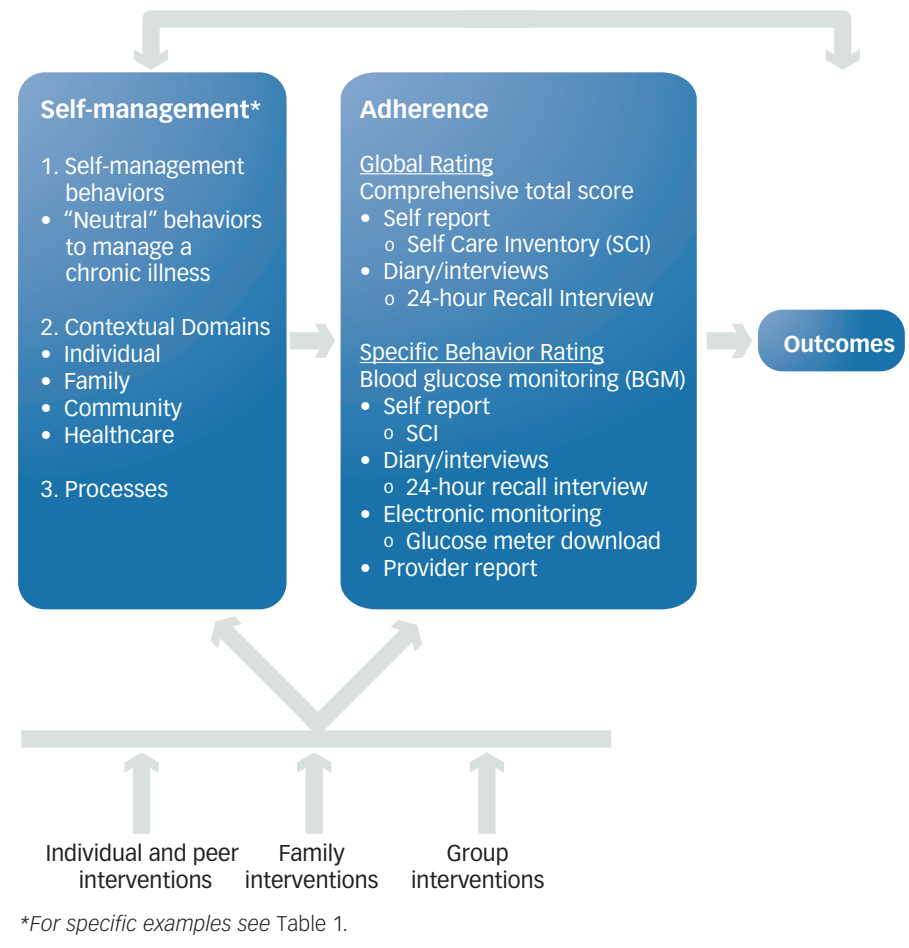

Regarding parental involvement and collaboration, some researchers have found that increased parental involvement is associated with more conflict among parents and youth, which then may decrease the youths' willingness to adhere to their diabetes regimen. ${ }^{6}$ However, the general consensus in the literature appears to be that parent involvement and collaboration between parent and youth in diabetes tasks have been found to be compensatory and significantly predict improved adherence to the diabetes regimen in general, especially if this collaborative involvement comes from the primary diabetes caregiver. ${ }^{7-10}$ Higher levels of parental readiness to change the balance of responsibility of diabetes care from parent to youth has also been shown to be related to more youth diabetes responsibility and self-efficacy (i.e., the perceived ability to manage diabetes care) as well as decreased general parental stress. ${ }^{11}$ Some researchers have suggested that too much independent responsibility for diabetes tasks in youth with type 1 diabetes may compromise diabetes self-care, even if it promotes maturity. ${ }^{12}$ Therefore, the transfer of this care needs to be conducted in a way that supports the youth's autonomy, without decreasing one's adherence. ${ }^{13,14}$ There is also evidence that increased positive family communication as well as high levels of diabetes knowledge are helpful in minimizing parent-youth verbal conflict regarding diabetes care. ${ }^{15}$

\section{Contextual Variables}

Four types of contextual variables are systems (i.e., individual, family, community, and healthcare system) that influence the youth and impact their self-management. These variables can be either modifiable or non-modifiable and are outlined below.
Individual

Individual characteristics that have been widely examined include youth demographics, cognitive abilities, psychological functioning, personality/coping style characteristics, and disease-specific characteristics. Considering youth demographics, research consistently shows that age is associated with adherence, where older youth are often found to have poorer adherence to their diabetes regimen. ${ }^{16,17}$ More research is emerging, which demonstrates that one's cognitive abilities, as measured by executive functioning (i.e., the ability to regulate behavior, metacognition, and cognitive autonomy) also play a role in successful diabetes self-care, beyond the influence of youth age..$^{16,18}$ Diabetes numeracy, the numerical skills needed to complete diabetes self-management tasks, has also been found to be related to improved diabetes knowledge, self-efficacy, and glycemic control in adults, ${ }^{19}$ yet this body of research needs to be extended to youth with type 1 diabetes.

Concerning psychological functioning, both internalizing (e.g., social anxiety and depression) and externalizing (e.g., acting out behaviors) problems are significant contributors to adherence across genders. ${ }^{20-22}$ In particular, there is evidence that symptoms of depression and anxiety may disrupt adherence through decreased concentration, impact on judgment ability, memory impairment, and decreased motivation and energy, which are all symptoms of the disorders themselves. ${ }^{23-25}$ Additional psychological characteristics that have been found to be related to diabetes adherence and subsequent glycemic control are maladaptive eating attitudes and behaviors, including the potential for insulin omission for the purposes of weight control. ${ }^{26}$

The relationships among certain personality characteristics (e.g., conscientiousness, extroversion, neuroticism, impulsivity, and assertiveness), individual coping styles (e.g., stress management), and diabetes management have also been examined..$^{27,28}$ Specifically, high youth conscientiousness and extraversion are associated with higher rates of completing diabetes cares, while high neuroticism and low conscientiousness are predictive of decreased adherence and a need for increased monitoring by parents throughout adolescence. ${ }^{28}$ Researchers found that youth's general stress impacts their adherence, especially for completing blood glucose monitoring (BGM), as the endorsement of high levels of general stress is indicative that youth may be experiencing stress regarding health, finances, living situations, parents, siblings, school, and friends. ${ }^{27}$

There are also disease-specific factors, including length of time since type 1 diabetes diagnosis, that also seem to impact adherence to diabetes self-management tasks, but these findings are more equivocal. Some researchers suggest that youth with a longer duration of diagnosis feel more comfortable with BGM and are more at ease with doing their diabetes care in public, ${ }^{29}$ while other studies have found that longer duration of diabetes is related to lower self-reported adherence rate ${ }^{27,30}$ and diabetes 'burnout'. ${ }^{31}$ Researchers have looked at the interrelationships of multiple variables that may also impact the association between length of time since diagnosis and adherence. They found that when these covariates are examined, age moderates any gender differences that were previously found in adherence, and the effects of duration of diabetes on adherence also appear to be mediated by youths' depressive symptoms. ${ }^{21}$ Therefore, diabetes duration may have both a positive and negative impact 
Table 1: Examples of Self-management Factors in Youth with Type 1 Diabetes

\begin{tabular}{|c|c|c|}
\hline Self-management Behaviors & $\begin{array}{l}\text { Contextual Variables } \\
\text { (Modifiable and Non-modifiable) }\end{array}$ & Processes \\
\hline Parental involvement/collaboration & Individual: & Cognitive: \\
\hline Division of diabetes responsibility & Age & Health beliefs \\
\hline Transfer of diabetes care & Executive functioning & Compensatory beliefs \\
\hline \multirow[t]{13}{*}{ Parent-youth communication } & Youth anxiety/depression & \\
\hline & Externalizing behaviors & \\
\hline & Stress & \\
\hline & Family: & Emotional: \\
\hline & Household organization/chaos & Threat to well-being \\
\hline & Spousal/partner support & Self-efficacy \\
\hline & Distribution of parental roles & \\
\hline & Maternal anxiety/depression & \\
\hline & Community: & Social: \\
\hline & Teacher victimization & Decision making skills \\
\hline & Peer pressure/bullying & Social attributions \\
\hline & Healthcare: & \\
\hline & Provider-patient relationship & \\
\hline
\end{tabular}

on diabetes care, and other factors may be even more relevant to overall adherence in youth with type 1 diabetes.

\section{Family}

The research with youth with type 1 diabetes related to the second contextual variable, the family system, has focused on examining multiple characteristics, such as socio-economic status (SES), ethnicity, family structure and organization, spousal/partner support, distribution of parental responsibilities, and parental psychological functioning. Specifically, families with heightened financial strain had poorer treatment outcomes and adherence. ${ }^{32,33}$ In a study of Hispanic youth with type 1 diabetes, those who had more recent generational status (i.e., their parents had immigrated more recently) in the US had better adherence. The authors hypothesize that those who are less acculturated may have greater respect for medical staff, which may be associated with better adherence. ${ }^{13}$ Researchers examined the relationship between adherence and a number of family structure variables, including maternal employment status, while controlling for the influence of youth age, pubertal status, SES, duration of diabetes diagnosis, gender, and ethnicity. They found no differences in self-care adherence for the family structure variables. ${ }^{34}$ The researchers did find that older age of youth and more advanced youth pubertal status were two variables associated with better adherence; therefore, they cautioned diabetes providers from stereotyping families based on their presenting family structure only (i.e., assuming that families from households where the mother is employed have worse adherence). ${ }^{16}$ Other researchers examined family organization and household chaos characteristics and found that high amounts of child routines in the household positively impact diabetes self-care. ${ }^{17}$

Both the amount of spousal/partner support felt by maternal caregivers and the division of parental responsibilities in caring for youth with type 1 diabetes have been found to be important. The literature suggests that maternal perceptions of support from her spouse/partner and involvement of paternal caregivers is beneficial to both maternal and youth functioning. ${ }^{35}$ Higher levels of paternal support is hypothesized to minimize maternal sense of helplessness and decrease diabetes-related verbal conflict between maternal caregivers and youth. ${ }^{36,37}$ Researchers have also identified that both maternal and paternal caregivers may have different, but equally important, roles in supporting youth's diabetes management. ${ }^{37,38}$ Specifically, maternal and paternal caregivers who have more positive relationships with their child and demonstrate regular monitoring also have youth with better adherence. ${ }^{38}$ Behavioral involvement in daily diabetes care tasks by maternal caregivers was associated with better adherence, whereas father behavioral involvement in daily diabetes care tasks was related to poorer adherence among youth with type 1 diabetes. ${ }^{38}$ Therefore, parenting behavior and gender are important to take into account when examining maternal and paternal roles in promoting diabetes adherence in youth with type 1 diabetes. ${ }^{37}$

One additional family factor that has been found to significantly impact adherence among youth with type 1 diabetes is maternal psychological functioning, including separation anxiety and depression. ${ }^{16,39}$ Maternal caregivers, who demonstrate difficulties with separation anxiety and attachment issues with their adolescents, are hypothesized to also have difficulties with their adolescent's eventual need for autonomy for diabetes care. ${ }^{16}$ Similarly, higher maternal depressive symptoms have been found to be associated with poorer youth diabetes adherence. ${ }^{39}$ Researchers suggested that the maternal depression symptoms may impede the ability of mothers to facilitate their youth's self-management skills over time. ${ }^{39}$

\section{Community}

Less research has been conducted regarding the third contextual variable, community, yet multiple studies have focused on the role of teachers and peers in impacting adherence among youth with type 1 diabetes. One study examined how negative (i.e., victimizing) teacher-youth interactions played a role in youth's diabetes adherence. They found that these challenging interactions decrease diabetes self-management behaviors for younger children, but not adolescents ages 12 and older. ${ }^{40}$ Negative peer influences have been shown to be detrimental to diabetes adherence among youth with type 1 diabetes, yet a strong parental foundation is thought to protect against this negative peer pressure. ${ }^{41}$ Youth who have 
reported being bullied or teased about type 1 diabetes have poorer adherence, especially for the more public and observable diabetes tasks (e.g., BGM and insulin administration). ${ }^{42}$ However, other researchers have attempted to determine whether certain peer groups can promote positive peer influences and have found that training in diabetes support among peers is helpful to building social support for youth with type 1 diabetes. ${ }^{43,44}$ Diabetes camps are also a community resource for youth with type 1 diabetes and future research is warranted to determine if diabetes camps serve as a potential protective factor for adherence.

\section{Healthcare System}

The final contextual variable proposed in the Modi et al. model considers the role of the healthcare system. ${ }^{5}$ Research in this area often focuses on examining the patient-provider relationship and communication. More patient-centered communication by the physician has been found to be related to better adherence and metabolic control. ${ }^{45}$ Youth's perception of support from their diabetes physicians and nurses has been found to be influential in helping patients share their concerns and be honest with medical providers about their diabetes, which ultimately helps improve diabetes adherence..$^{30}$ It is important for healthcare systems (i.e., diabetes clinics) to determine effective ways of assessing self-management behaviors as well as the contextual factors (e.g., individual and family systems) in routine clinical visits. For example, the assessment of psychological characteristics, such as depressive symptoms, among youth with type 1 diabetes should be integrated into the diabetes clinic visits using well-validated measures. ${ }^{25}$

\section{Processes}

The third component to Modi et al.'s self-management model examines the processes that influence how the self-management behaviors and contextual variables interact. ${ }^{5}$ Researchers have attempted to understand how different individual perceptions of the world (e.g., cognitive, emotional, and social processes) link self-management and contextual variables.

The Health Belief Model (HBM) describes the potential influence of cognitive processes, such as one's perceptions of how certain behaviors do or do not have the potential to impact health outcomes. ${ }^{46}$ The HBM findings are mixed. ${ }^{9,29,47}$ Some researchers describe the importance of this model in understanding youth's health locus of control and how it may produce better health outcomes. ${ }^{47}$ Others do not find the HBM to be adequate as it does not take into account other potentially influential psychosocial factors, ${ }^{9}$ and it has not been predictive of diabetes adherence. ${ }^{29}$ The use of compensatory beliefs, or rationalizing that the negative effects of one behavior (e.g., not doing BGM) can be counteracted by other compensatory behaviors (e.g., skipping breakfast), have also been examined. These maladaptive convictions are hypothesized to alleviate any mental conflict over engaging in behaviors that are at odds with one's diabetes regimen recommendations, and they have been shown to be predictive of poorer BGM adherence in youth with type 1 diabetes. ${ }^{48}$

Research has also explored how emotional processes may influence adherence among youth with type 1 diabetes. Specifically, researchers found youth's views about diabetes and its treatment as a threat to emotional well-being. Youth perceptions of threats to emotional well-being was the best predictor of adherence when compared to other variables, such as physician and nurse support, motivation to comply, energy to complete the tasks, and views of diabetes as a threat to physical well-being. ${ }^{30}$ Youth who perceived diabetes as a threat to emotional well-being were 7.68 times more likely to complete their diabetes care tasks, than those who did not hold those views. ${ }^{30}$ Researchers are not endorsing the use of 'scare tactics' to increase the perception of diabetes as a threat to youth with type 1 diabetes, but rather they suggest that efforts should be made to instill a sense of higher self-efficacy in being able to manage their health through increased maternal empathy and perceived support from diabetes physicians and nurses. It is anticipated that these beliefs will then promote motivation, energy, and willpower to improve health outcomes for youth with type 1 diabetes. ${ }^{30,49}$

Two social processes that have been examined among youth with type 1 diabetes include youth's acquisition of decision-making skills from their family of origin $^{50}$ and the impact of negative social attributions of peers. ${ }^{51}$ Specifically, researchers found that negative family communication resulted in youth with type 1 diabetes observing the family decision making process as pessimistic. Youth who experienced negative family communication also lacked the ability to take responsibility for their own behaviors, and they were less able to take others' perspectives. It is likely that all of these characteristics impacted how diabetes responsibility and management were allocated and executed in the family. ${ }^{50}$ The social information processing model outlines how youth with type 1 diabetes, who engage in higher amounts of negative attributions, misinterpret that peers will react negatively if they observed youth completing diabetes tasks. More negative assumptions have been found to be associated with poorer adherence, especially when adolescents are in social situations. ${ }^{51}$

\section{Adherence}

Adherence is often defined as, "The extent to which a person's behavior (in terms of taking medications, following diets, or executing lifestyle changes) coincides with medical or health advice" ${ }^{52}$ Despite the relative consensus on a definition of adherence, there are often different approaches to operationally assessing and measuring adherence. ${ }^{53}$ Some researchers focus on global, comprehensive assessments of adherence, while other focus on specific adherence tasks separately. Therefore, the how, when, where, and by whom these adherence behaviors are assessed, result in different ratings of degrees of medical adherence. ${ }^{53}$ It is recommended that adherence measures should be continuous, dynamic, and capture specific regimen behaviors relevant to the disease population. ${ }^{54}$

\section{Diabetes-specific Adherence}

Similar to youth with other chronic medical illnesses, youth with type 1 diabetes have been shown to have difficulties with adherence to their medical regimen. Assessment of diabetes regimen adherence has included both measures that have been used to provide global information about adherence (e.g., summary scores from self report measures) as well as measures that provide information about specific adherence behaviors (e.g., BGM). Thus, both the multidimensional nature of the diabetes regimen as well as the type of adherence assessment method used is important for researchers and clinicians to consider. Recent work by Quittner et al. ${ }^{1}$ and Rapoff ${ }^{55}$ examined adherence assessments that were related to the individual's execution of the recommended medical 
regimen for pediatric patients with a wide variety of chronic diseases, including type 1 diabetes. Quittner et al. reviewed three different accepted methods of assessing diabetes adherence behaviors in youth: self-report measures [e.g., Self Care Inventory (SCI)], diary/interview measures (e.g., 24-hour Recall Interview), and electronic monitoring (e.g., blood glucose meter download). ${ }^{55}$ In general, Quittner et al. recommended that one additional assessment measure be utilized along with an electronic monitoring measure for all adherence research. A more thorough review of the strengths and weaknesses of each method of assessing diabetes adherence is beyond the scope of this review and is summarized fully in Quittner et al. ${ }^{55}$

Although diabetes regimen adherence has been measured using different methods (e.g., self-report measures, diary/interview measures, and electronic monitoring), there is empirical evidence demonstrating the associations between many of these adherence measures and glycemic control. ${ }^{26,56}$ There are also well-established relationships between global measures of adherence (e.g., SCI) ${ }^{55}$ as well as specific diabetes adherence behaviors (e.g., BGM) and glycemic control. BGM is the one specific diabetes regimen adherence behavior that has been repeatedly found to be a primary determinant of glycemic control..$^{57-60}$ The assessment methodologies utilized for assessing BGM in these studies are varied (e.g., self-report, diary/interview, meter downloads, and/or provider ratings), and they are all still fraught with concerns about reliability and validity. ${ }^{55,61}$ Despite this lack of consensus on how to best assess BGM, BGM is still often utilized as an indicator of a patient's overall adherence to one's diabetes-specific medical regimen. ${ }^{56}$

\section{Diabetes-specific Interventions}

As researchers have continued to identify the self-management characteristics that impact adherence, a variety of psychological interventions have sought to address modifiable characteristics. The next section will review the efforts of individual and peer, family, group, and the combination of family and group interventions in addressing self-management, adherence, and outcomes for youth with type 1 diabetes.

\section{Individual and Peer Interventions}

As outlined by wysocki et al., there have been many individual and family interventions that focus on behavioral management, communication and problem-solving, as well as stress, coping, and psychological adjustment. ${ }^{4}$ In addition, there have been a number of interventions that have targeted peers and social interactions for youth with type 1 diabetes. ${ }^{44,62}$ Researchers found that participant support from a best friend impacted youths' adjustment to type 1 diabetes by increasing their diabetes knowledge and social support for their diabetes care. ${ }^{44}$ Similarly, preliminary analyses examining an intervention which targeted retraining negative social attributions in youth with type 1 diabetes demonstrated that problem-solving strategies were helpful in improving youths' reported adherence in social situations. ${ }^{62}$

\section{Family Interventions}

Family-based interventions, such as multisystemic therapy (MST) ${ }^{63}$ and behavioral family systems therapy (BFST), ${ }^{64}$ have been used to help improve diabetes management in youth. MST family-based interventions are conducted in youths' homes with their family members and have been found to positively impact both adherence to frequency of BGM recommendations as well as health (i.e., metabolic control) and individual (i.e., inpatient admissions) outcomes, especially among those who chronically evidence poor diabetes control. Researchers found that family-based interventions using BFST showed improvement in self-management behaviors (i.e., parent-adolescent relationship and diabetes-specific conflict) as well as a health outcome (i.e., glycated hemoglobin $\left[\mathrm{HbA}_{1 \mathrm{c}}\right]$ ) as compared with a randomized education group or a current therapy group..$^{64,65}$ However, results of treatment, in terms of improving psychological functioning, varied by contextual variables (e.g., age and gender), which indicate a need for more research on the effectiveness of family-based interventions with males and females of various ages. ${ }^{64,65}$ Moreover, those participating in this family treatment did not report changes in adherence behaviors to their medical regimens. Therefore, it also important to see if certain self-management interventions can impact outcomes without being mediated by adherence, similar to what Modi et al. suggest in their proposed model.

\section{Group Interventions}

Group interventions for youth with type 1 diabetes, which focus on providing peer support and developing problem-solving skills, have been able to improve outcomes, such as $\mathrm{HbA}_{1 \mathrm{c}}$ levels $\mathrm{s}^{66,67}$ and quality of life (QOL) for those with insulin pumps. ${ }^{68,69}$ Stress management and coping skills training have impacted other outcomes, such as reduced diabetes-related stress ${ }^{70}$ and improved social interactions. ${ }^{71}$

\section{Combined Group and Family Interventions}

Peer and family-based group therapies have been typically used as separate interventions with youth with type 1 diabetes. Opipari-Arrigan et al. developed an intervention, which combines both family and peer group intervention strategies, by offering separate, but parallel, youth and parent groups. ${ }^{72}$ This group therapy intervention demonstrated improved readiness to change the balance of responsibility of diabetes care as reported by parents as well as an increase in parental involvement in the division of diabetes responsibility as reported by both the parents and youths. ${ }^{72}$

In summary, many of these psychological interventions focused on behavioral factors that can be categorized as either self-management or adherence behaviors. These interventions targeted not only improved health outcomes, such as $\mathrm{HbA}_{1 \mathrm{c}}$, but a variety of other outcome variables. Future research will need to continue to determine which outcome variables are important to target, in addition to the more traditional health outcome of $\mathrm{HbA}_{1 \mathrm{c}}$, which is often resistant to clinically significant change in behavioral health interventions.

\section{Future Implications and Recommendations}

It has been well established that $\mathrm{HbA}_{1 \mathrm{c}}$ is one of the primary factors impacting long-term complications for individuals with diabetes ${ }^{73}$ and is often considered the hallmark health outcome variable in research involving youth with type 1 diabetes. In addition to traditional health outcomes in chronic medical illnesses, the Modi et al. model outlines other individual (e.g., quality of life, school absences, and healthcare utilization) and system (e.g., treatment efficacy, clinical decision making, and healthcare delivery) outcomes that are also important to understand. ${ }^{5}$ Researchers have explored several of these other individual and system 
outcomes, which are also influenced by self-management and adherence factors in youth with type 1 diabetes, but more research on these outcomes is warranted. An additional outcome measure that should be more fully explored is cost savings associated with an intervention. ${ }^{74}$ Little attention has been paid to intervention cost-effectiveness and very few investigations have systematically examined how to translate research-based interventions into clinical settings, especially longitudinally. ${ }^{75}$

The Modi et al. model ${ }^{5}$ is an empirically-derived model for pediatric chronic medical illness, in general, and includes an examination of self-management, adherence, and outcomes. This model has utility for organizing the large amount of behavioral health research on youth with type 1 diabetes; however, some questions remain to be examined empirically. For example, given the nature of the diabetes-specific medical regimen and its link to glycemic control, how does adherence act as a mediating variable between self-management and health outcomes? Similarly, are there some self-management factors that can impact outcomes without adherence as a mediating variable? Researchers examining behavioral factors in youth with type 1 diabetes would benefit from framing future descriptions of their studies within the context of the Modi et al. model, which distinguishes between interventions that focus on self-management versus adherence (or both) to help understand how they impact health outcomes. ${ }^{5}$ As more children of younger ages are diagnosed with type 1 diabetes, researchers will need to expand the body of literature to understand the self-management, adherence, and outcomes for young children with type 1 diabetes. Modi et al.'s model can help drive the development and evaluation of empirically-supported interventions to modify diabetes-related behavioral factors at different developmental stages to improve diabetes outcomes, ultimately preventing complications and mortality in youth with type 1 diabetes.
1. Rapoff MA, Adherence to Pediatric Medical Regimens, 2nd edition, New York, NY: Springer, 2010.

2. Kovacs $\mathrm{M}$, Goldsteon $\mathrm{D}$, Obrosky SD, Iyengar S, Prevalence of predictors of pervasive noncompliance with medical treatment among youths with insulin dependent diabetes mellitus, I Am Acad Child PSY, 1992:31:1112-9.

3. Wysocki T, Buckloh LM, Lochrie AS, Antal H, The psychologic context of pediatric diabetes, Pediatr Clin N Am, 2005;52:1755-78.

4. Wysocki T, Buckloh LM, Greco P, The psychological context of diabetes mellitus in youths, In: Roberts MC, Steele RG (eds), Handbook of Pediatric Psychology, 4th edition, New York: The Guilford Press.

5. Modi AC, Pai A, Hommel K, et al., Pediatric self-management: a framework for research, practice, and policy, Pediatrics, 2012;129:e473-85

6. Lewin $A B$, Heidgerken $A D$, Geffken $G R$, et al., The relation between family factors and metabolic control: the role of diabetes adherence, J Pediatr Psychol, 2006:31:174-83.

7. Anderson BJ, Vangness L, Connell A, et al., Family conflict, adherence, and glycaemic control in youth with short duration type 1 diabetes, Diabetic Med, 2002;19:635-42.

8. Berg CA, Schindler I, Maharajh S, Adolescents' and mothers' perceptions of the cognitive and relational functions of collaboration and adjustment in dealing with type 1 diabetes, J Fam Psychol, 2008:22:865-74

9. Gillibrand R, Stevenson J, The extended health belief model applied to the experience of diabetes in young people, Brit J Health Psych, 2006;11:155-69.

10. Wysocki T, Nansel TR, Holmbeck GN, et al., Collaborative involvement of primary and secondary caregivers: associations with youths' diabetes outcomes, J Pediatr Psychol, 2009:34:869-81.

11. Kaugars A, Kichler J, Alemzadeh R, Assessing readiness to change the balance of responsibility for managing type 1 diabetes mellitus: adolescent, mother, and father perspectives, Pediatr Diabetes, 2011;12:547-55.

12. Miller VA, Drotar DD, Discrepancies between mother and adolescent perceptions of diabetes-related decision-making autonomy and their relationship to diabetes-related conflict and adherence to treatment, J Pediatr Psychol, 2003:28:265-74.

13. Hsin O, La Greca AM, Valenzuela J, et al., Adherence and glycemic control among Hispanic youth with type 1 diabetes: role of family involvement and acculturation, I Pediatr Psychol, 2010;35:156-66.

14. Wolpert HA, Anderson BJ, Weissberg-Benchell, Transitions in care: meeting the challenges of type 1 diabetes in young adults, Alexandria, VA: American Diabetes Association, 2009.

15. Wysocki T, Lochrie A, Antal H, Buckloh LM, Youth and parent knowledge and communication about major complications of type 1 diabetes, Diabetes Care, 2011;34:1701-5.

16. Dashiff $\mathrm{C}$, Vance $\mathrm{D}$, Abdullatif $\mathrm{H}$, Wallander J, Parenting, autonomy and self-care of adolescents with type 1 diabetes, Child Care HIth Dev, 2009;35:79-88.

17. Greening L, Stoppelbein L, Konishi C, et al., Child routines and youths' adherence to treatment for type 1 diabetes, J Pediatr Psychol, 2007;32:437-47.

18. Bagner DM, Williams LB, Geffken GR, et al., Type 1 diabetes in youth: the relationship between adherence and executive functioning, Child Health Care, 2007;36:169-79.

19. Cavanaugh $K$, Huizinga MM, Wallston KA, et al., Association of numeracy and diabetes control, Ann Intern Med, 2008; 148:737-46.

20. Di Battista AM, Hart TA, Greco L, Gloizer J, Type 1 diabetes among adolescents: reduced diabetes self-care caused by social fear and fear of hypoglycemia, Diabetes Educator 2009;35:465-75.
21. Korbel $C D$, Wiebe DJ, Berg CA, Palmer D, Gender differences in adherence to type 1 diabetes management across adolescence: the mediating role of depression, Child Health Care, 2007;36:83-98.

22. Naar-King $\mathrm{S}$, Idalski $\mathrm{A}$, Ellis $\mathrm{D}$, et al., Gender differences in adherence and metabolic control in urban youth with poorly controlled type 1 diabetes: the mediating role of mental health symptoms, J Pediatr Psychol, 2006;31:793-802.

23. Herzer M, Hood KK, Anxiety symptoms in adolescents with type 1 diabetes: association with blood glucose monitoring and glycemic control, J Pediatr Psychol, 2010;35:415-25.

24. Hilliard ME, Herzer M, Dolan LM, Hood KK, Psychological screening in adolescents with type 1 diabetes predicts outcomes one year later, Diabetes Res Clin Pr, 2011:94:39-44.

25. McGrady ME, Hood KK, Depressive symptoms in adolescents with type 1 diabetes: associations with longitudinal outcomes, Diabetes Res Clin Pr, 2010;88:e35-7.

26. Kichler JC, Foster C, Opipari-Arrigan L, The relationship between negative communication and body image dissatisfaction in adolescent females with type 1 diabetes mellitus, J Health Psychol, 2008:13:336-47.

27. Farrell SP, Hains AA, Davies WH, et al., The impact of cognitive distortions, stress, and adherence on metabolic control in youths with type 1 diabetes, I Adolescent Health, 2004;34:461-7

28. Wheeler K, Wagaman A, McCord D, Personality traits as predictors of adherence in adolescents with type 1 diabetes, J Child Adolesc Psychiatr Nurs, 2012;25:66-74.

29. Patino AM, Sanchez J, Eidson M, Delamater AM, Health beliefs and regimen adherence in minority adolescents with type 1 diabetes, J Pediatr Psychol, 2005:30:503-12.

30. Kyngas HA, Predictors of good adherence of adolescents with diabetes (insulin-dependent diabetes mellitus), Chronic Illness, 2007:3:20-8.

31. Polonsky WH, Diabetes Burnout: What To Do When You Can't Take It Anymore, Alexandria, VA: American Diabetes Association, 1999

32. Drew LM, Berg C, King P, et al., Depleted parental psychological resources as mediators of the association of income with adherence and metabolic control, J Fam Psychol, 2011;25:751-8.

33. Pereira MG, Berg-Cross L, Almeida P, Machado JC, Impact of family environment and support on adherence, metabolic control, and quality of life in adolescents with diabetes, Int J Behav Med, 2008:15:187-93.

34. Dashiff $\mathrm{C}$, Bartolucci A, Wallander J, Abdullatif $\mathrm{H}$, The relationship of family structure, maternal employment, and family conflict with self-care adherence of adolescents with type 1 diabetes, Fam Syst Health, 2005;23:66-79.

35. Lewandowski A, Drotar D, The relationship between parent-reported social support and adherence to medical treatment in families of adolescents with type 1 diabetes, J Pediatr Pychol, 2007;32:427-36.

36. Hansen JA, Weissbrod C, Schwartz DD, Taylor WP, Paternal involvement in pediatric type 1 diabetes: fathers' and mothers' psychological functioning and disease management Fam Syst Health, 2012;30:47-59.

37. Shorer M, David R, Schoenberg-Taz M, et al., Role of parenting style in achieving metabolic control in adolescents with type 1 diabetes, Diabetes Care, 2011:34:1735-7.

38. Palmer DL, Osborn P, King PS, et al., The structure of parental involvement and relations to disease management for youth with type 1 diabetes, J Pediatr Psychol, 2011;36:596-605

39. Wiebe DJ, Gelfand D, Butler JM, et al., Longitudinal associations of maternal depressive symptoms, maternal involvement, and diabetes management across adolescence, J Pediatr Psychol, 2011;36:837-46.
40. Peters $C D$, Storch EA, Geffken GR, et al., Victimization of youth with type-1 diabetes by teachers: relations with adherence and metabolic control, I Child Health Care, 2008;12:209-20.

41. Drew LM, Berg C, Wiebe DJ, The mediating role of extreme peer orientation in the relationships between adolescent-parent relationship and diabetes management, J Fam Psychol, 2010;24:299-306.

42. Storch EA, Heidgerken AD, Geffken GR, et al., Bullying, regimen self-management, and metabolic control in youth with type I diabetes, J Pediatr, 2006;148:784-7.

43. Hains AA, Berlin KS, Davies WH, et al., Attributions of adolescents with type 1 diabetes related to performing diabetes care around friends and peers: the moderating role of friend support, J Pediatr Psychol, 2007;32:561-70.

44. Greco P, Pendley JS, MCDonell K, Reeves G, A peer group intervention for adolescents with type 1 diabetes and their best friends, J Pediatr Psychol, 2001:26:485-90.

45. Croom A, Wiebe DJ, Berg CA, et al., Adolescent and parent perceptions of patient-centered communication while managing type 1 diabetes, J Pediatr Psychol, 2011;36:206-15

46. Becker $\mathrm{MH}$, The health belief model and personal health behavior, Health Edu Man, 1974;2:324-473.

47. Nabors L, MCGrady M, Kichler J, Children's attitudes toward their diabetes, locus of control, and HbA1c levels, J Dev Disabil, 2010;22:475-84.

48. Rabiau MA, Knauper B, Nguyen TK, et al., Compensatory beliefs about glucose testing are associated with low adherence to treatment and poor metabolic control in adolescents with type 1 diabetes, Health Educ Res, 2009;24:890-6.

49. Lloyd SM, Cantell M, Pacaud D, et al., Brief report: hope, perceived maternal empathy, medical regimen adherence, and glycemic control in adolescents with type 1 diabetes, J Pediatr Psychol, 2009;34:1025-9.

50. Miller VA, Drotar D, Decision-making competence and adherence to treatment in adolescents with diabetes, J Pediatr Psychol, 2007;32:178-88.

51. Hains AA, Berlin KS, Davies WH, et al., Attributions of adolescents with type 1 diabetes in social situations: relationship with expected adherence, diabetes stress, and metabolic control, Diabetes Care, 2006;29:818-22.

52. Haynes R, Introduction, In: Haynes R, Taylor D, Sackett D (eds), Compliance in Health Care, Maryland: Johns Hopkins University Press, 1979;1-7.

53. Johnson SB, Carlson DN, Medical regimen adherence: concepts, assessment, and interventions, In: Raczynski JM, Leviton LC (eds.), Handbook of Clinical Health Psychology, Volume 2: Disorders of Behavior and Health, Washington, DC: American Psychological Association Press; 2004;329-354.

54. Johnson SB, Measurement and detection of treatment non-adherence in the adolescent, Proceedings of the National Institute of Diabetes and Digestive and Kidney Diseases National Institutes of Health on Non-Adherence in Adolescent with Chronic Illness, 2008 September, Bethesda, MD, 2008.

55. Quittner AL, Modi AC, Lemanek C, et al., Evidence-based assessment of adherence to medical treatments in pediatric psychology, J Pediatr Psychol, 2008;33:916-36.

56. Hood KK, Peterson CM, Rohan JM, Drotar D, Association between adherence and glycemic control in pediatric type 1 diabetes: a meta-analysis, Pediatrics, 2009;124:1171-9.

57. Anderson B, Ho J, Brackett J, et al., Parental involvement in diabetes management tasks: relationships to blood glucose monitoring adherence and metabolic control in young adolescents with insulin-dependent diabetes mellitus, J Pediatr, 1997;130:257-65. 
58. Hood KK, Butler DA, Volkening LK, et al., The blood glucose monitoring communication questionnaire: an instrument to measure affect specific to blood glucose monitoring, Diabetes Care, 2004;27:2610-5.

59. Levine B, Anderson B, Butler D, et al., Predictors of glycemic control and short-term adverse outcomes in youth with type 1 diabetes, J Pediatr, 2001;139:197-203.

60. Lewin AB, La Greca AM, Geffken GR, et al., Validity and reliability of an adolescent and parent rating scale of type diabetes adherence behaviors: the Self-Care Inventory (SCI), J Pediatr Psychol, 2009;34:999-1007.

61. Guilfoyle SM, Crimmins NA, Hood KK, Blood glucose monitoring and glycemic control in adolescent with type 1 diabetes: meter downloads versus self-report, Pediatr Diabetes, 2011;12:560-6.

62. Salamon KS, Hains AA, Fleischman KM, et al., Improving adherence in social situations for adolescents with type 1 diabetes mellitus (T1DM): a pilot study, Primary Care Diabetes, 2010:4:47-55.

63. Ellis DA, Frey MA, Naar-King S, et al., Use of multisystemic therapy to improve regimen adherence among adolescents with type 1 diabetes in chronic poor metabolic control. A randomized control trial, Diabetes Care, 2005;28:1604-10.
64. Wysocki T, Harris M, Greco P, et al., Randomized, controlled trial of behavior therapy for families of adolescents with insulin-dependent diabetes mellitus, I Pediatr Psychol, 2000;25:23-33.

65. Wysocki T, Harris MA, Buckloh LM, et al., Randomized trial of behavioral outcomes in adolescents. Maintenance of effects on diabetes outcomes in adolescents, Diabetes Care, 2007;30:555-60.

66. Anderson BJ, Wolf MT, Burkhart RG, et al., Effects of peer-group intervention on metabolic control of adolescents with IDDM. Randomized outpatient study, Diabetes Care, 1989;12:179-83.

67. Kaplan RM, Chadwick MW, Schimmel LE, Social learning intervention to promote metabolic control in type I diabetes mellitus: pilot experiment results, Diabetes Care, 1985;8:152-5.

68. Boland EA, Grey M, Oesterle A, et al., Continuous subcutaneous insulin infusion: a new way to lower risk of severe hypoglycemia, improve metabolic control, and enhance coping in adolescents with type 1 diabetes, Diabetes Care, 1999;22:1779-84.

69. Grey M, Boland E, Davidson M, et al., short-term effects of coping skills training as adjunct to intensive therapy in adolescents, Diabetes Care,1998;21:902-8.
70. Boardway RH, Delamater AM, Tomakowsky J, Gutai JP, Stress management training for adolescents with diabetes, J Pediatr Psychol, 1993;18:29-45

71. Mendez FJ, Belendez, M, Effects of a behavioral intervention on treatment adherence and stress management in adolescents with IDDM, Diabetes Care, 1997;20:1370-5

72. Opipari-Arrigan L, Kichler J, Fredericks E, et al., Self-management intervention improved diabetes-related functioning in at-risk adolescents with type 1 diabetes [abstract], Presented at: American Diabetes Association 65th Scientific Sessions, San Diego CA, 2005

73. The DCCT Research Group, The effect of intensive treatment of diabetes on the development and progression of long-term complications in insulin-dependent diabetes mellitus, New Engl J Med, 1993;329:977-86.

74. Stark $\sqcup$, Mulvihil MM, Powers SW, et al., Behavioral intervention to improve calorie intake of children with cystic fibrosis: treatment versus wait list control, J Pediatr Gastr, 1996;22:240-53.

75. Delamater AM, Jacobson AM, Anderson B, et al., Psychosocia therapies in diabetes: report of the psychosocial therapies working group, Diabetes Care, 2001;24:1286-92. 